\title{
Deuteronomium und Deuteronomius.
}

Angeregt durch Nestle (vergl. diese Zeitschrift XVI, $325 \mathrm{ff}$., XVIII, 252 ff.) und Schulte (ebends. XVII, 18I f.), habe ich sämtliche Inkunabeln und alte Handschriften der hiesigen Stadtbibliothek auf die Übersichriften des 5. Buches Mosis hin angesehen. Die alten Drucke des katholischen Seminars wurden auf meine Bitten von liebenswürdiger Seite durchgesehen. Dieselben sind, soweit sie nicht auch auf der Stadtbibliothek vorhanden sind, mit dem Sigel [S] bezeichnet.

Ich gebe nun zuerst das vorhandenc Material:

I. Deuteronomium lesen folgende Drucke:

1) Biblia Latina, Basel (Froben) r49r.

2) Biblia integra, illustrata cum concordantiis, Basel (Froben) 1495.

3) Biblia Latina, Basel (Langendorff und Froben) I 509.

4) Biblia c. pleno apparatu, Basel (Froben) I5I4.

5) Biblia Latina, Basel (Richel) 1476 [S].

6) Biblia Latina, Basel (Richel) 1477 .

7) Biblia Latina, Basel (Ärberger) I 503 [S].

8) Biblia Latina, Basel (Kessler) 1487 [S].

9) Biblia cum concord., Lyon (Sacon, expensis Koberger) $15 \mathrm{I} 2$.

I0) Biblia c.concord., Lyon (Sacon, exp. Koberger) I 515.

II) Biblia c. concord., Lyon (Sacon, exp. Koberger) I 516.

I2) Biblia c. concord., Lyon (Mario, exp. Koberger) I 520. 
13) Biblia c. concord., Lyon (Moylin) 1520.

14) Biblia Latina, Venedig (Reynsburch) 1478.

15) Biblia Latina, Venedig (Jenson) 1479.

16) Biblia Latina, Venedig (Seligenstadt) I484.

17) Biblia Latina, Venedig (Paganus de Paganinis) I 501.

I8) Biblia c. concord., Venedig (Luc. de Giunta) 1519.

19) Biblia c. concord., Strassburg 1497 (bei Hain Repertor. Bibliogr. Stuttg. 1826 ff. unter Nr. 3122).

20) Biblia, sine loco, 1479 (Hain 3075).

2I) Biblia Sacra, s. 1., I48I (Hain 308I).

22) Biblia Latina, s. 1., 1482 (Hain 3086).

23) Biblia, pars I, s. 1.1483 (Hain 3088).

24) Biblia, s. 1. 1486 (Hain 3095) [S].

25) Biblia c. concord., s. 1.1489 (Hain 3104).

26) Biblia Sacra, s. 1. s. dato.

27) Biblia Latina, s. 1. s. d. (Hain 3I73).

28) Testamentum Vetus [Basel, Richel?] (Hain 304I).

Und last not least die

29) Biblia Latina, Mainz (Fust und Schöffer) I462.

II. Deuteronomius lesen folgende Drucke:

I) Biblia Latina,. Nürnberg (Koberger) I475; aber explicit Deuteronomium! (Hain 3056).

2) Biblia Latina, Nürnberg (Koberger) 1477 (explicit Deuteronomium!).

3) Biblia Latina, Nürnberg (Koberger) I478 (expl. Deuteronomium!).

4) Biblia Latina, Nürnberg (Koberger) I479 (expl. Deuteronomium!)

5) Biblia Latina, Nürnberg (Koberger) I482 (expl. Deuteronomium!)

6) Biblia Latina, s. 1. I486 (expl. Deteronomium; dazu hat jede Seite die Überschrift Deuteronomium, während die andern Inkunabeln, die als Titel 
Deuteronomius führen, so auch auf den Seiten lesen).

7) Biblia Latina, Köln (Homborch) I479 (expl. Deuteronomium!).

8) Biblia Latina, Ulm (Zeiner) 1480 (expl. Deuteronomium!) (Hain 3079).

9-II) Drei Bibelhandschriften der Vulgata aus dem XIV. Jahrhundert, die aber mit explicit Deuteronomius schliessen.

Es kommen nun die deutschen Bibeln an die Reihe:

I2) Bibel, Augsburg (nach Hain 3I34 Günther Zeiner) 1477.[S] - Anfang: „Buch deutronomii i. e. der andern ee" ( $=$ ehe = Bund). Schluss: „hie hat eyn end das buch deutronomius."

13) Bibel, teutsch, Augsburg (Anton Sorg) I480: „hie hebet an das buch deutronomii", „hie hat ein end das buch deutronomius" (Hain 3136).

I4) Bibel, Augsburg (Hans Schönsperger) I487: „hie hat ein ende das buch deutronomius" (Anfang fehlt) (Hain 3 I39).

I5) Bibel, teuitsch, Augsburg (H. Otmar) I 507 : ,hebt an das buch deuteronomij", "hie hat ain end das buch deuteronomius".

16) Biblia, d. i. die gantze Hailige Schrifft Teutsch, Nürnberg (Koberger) 1483 [S]: „hebt an das buch Deutronomii“, „hie hat ein ende $d z$ buch Deutronomius" (Hain 3137).

17) Biblia Dudesch, Halberstadt (Trutebul) I $520: \cdot$,hijr heuet sick an dat vefte bök Moyse, genoemet Deutronomius".

III. Interessant sind die Bibeldrucke, die mit dem Kommentar des Lyra versehen sind: 
a) Text und Postille haben Deuteronomium in folgenden Ausgaben:

I) Biblia, pars I. c. glossa N. de Lyra, Basel (Froben) I 50 I.

2) Biblia c. post. Lyrae, Basel (Amerbach und de Langen) 1502.

3) Biblia c. post. Lyrae, Nürnberg (Koberger) 1497 [S].

b) Text und Postille haben Deuteronomius:

I) Biblia sacra c. comment. [Nürnberg, 1485?]; aber expl. Deuteronomium! (Hain 3166).

c) Im Text findet sich Deuteronomium, in der Postille Deuteronomius:

I) Biblia, pars I. c. glossa, Basel (Langendorff u. Froben) 1498.

2) Biblia Tota c. comm., Venedig (Renner deHailbrun) I482 (Hain 3165).

d) Desgleichen hat eine Incunabel des Lyra, pars I. tract. super toto corpore Bibl. [Strassburg 1492?] ,incipit liber Deuteronomius“, während im Text wieder Deuteronomium steht.

IV. Aus dem zusammengebrachten Material, das der Ergänzung aus anderen Büchereien bedarf, scheint sich mir nun Folgendes zu ergeben:

I) Wenn auch in alten Drucken das 5. Buch Mosis gewöhnlich als Deuteronomium bezeichnet wird, so ist doch die Benennung Deuteronomius nicht allzu selten.

2) Die verschiedenen Bezeichnungen in den lateinischen Bibeln verteilen sich auf verschiedene Druckorte und Drucker. So haben die Koberger Drucke von Nürnberg ausser III a. $3^{x}$ stets Deuteronomius, während die auf seine Kosten in Lugdunum hergestellten Exemplare die landläufige Form aufweisen.

I III a. 3 [S] ist von mir nicht persönlich eingesehen worden; ich muss hier der gütigen Mittheilung vertrauen. 
3) Die deutschen Übersetzungen, sowie die lateinischen HSS. des XIV. Jahrh. - soweit ich sie kenne - haben Deuteronomius.

4) In derselben Ausgabe wechselt Deuteronomius mit Deuteronomium (Text und Postille; stets: explicit Deuteronomium, auch wenn Anfang und Seitenüberschrift Deuteronomius lauten).

5) Alles das zeigt uns, dass die beiden Formen promiscue gebraucht worden sind.

6) Die Form Deuteronomium lehnt sich an das Griechische

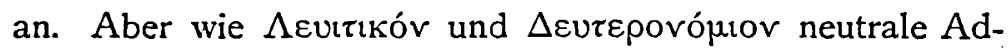
jektive sind, die als Ergänzung unbedingt ein. Bıß íov verlangen, so sind Leviticus und Deuteronomius männliche Adjektive, deren jeweilige Ergänzung liber ist. Vielleicht kommt Deuteronomium erst wieder mit dem Bekanntwerden des griech. Textes auf. Keiner stösst sich heute an dem mask. Leviticus; ihm ist der Sieg besser gelungen als dem Deuteronomius, das aus „alter Bücher Staub“ hervorgeholt zu haben, Nestles Verdienst ist.

Mainz, d. I8. Oktober 1898.

A. Freiherr v. Gall. 